\title{
49. Mathematical solutions for the flexural analysis of Mindlin's first order shear deformable circular plates
}

\author{
Charles Chinwuba Ike \\ Department of Civil Engineering, Enugu State University of Science and Technology, Enugu State, Nigeria \\ E-mail: charles.ike@esut.edu.ng
}

Received 15 March 2018; received in revised form 20 April 2018; accepted 7 May 2018 DOI https://doi.org/10.21595/mme.2018.19825

Check for updates

Copyright $(\mathbb{C} 2018$ Charles Chinwuba Ike. This is an open access article distributed under the Creative Commons Attribution License, which permits unrestricted use, distribution, and reproduction in any medium, provided the original work is properly cited.

\begin{abstract}
In this work, the problem of first order shear deformable solid circular plate under transverse load was solved mathematically. The problem considered was assumed axisymmetric. The plate and loading were considered axisymmetric. The problem was defined as a boundary value problem of a system of differential equations of equilibrium in terms of the stress resultants and the stress - resultants - displacement relations. The set of equations were considered simultaneously to express them in variable separable form. The mathematical technique of separation of variables was then used to obtain solutions for the unknown generalised displacements. Specific problems of clamped edge plates and simply supported edge plates under uniformly distributed load and point load at the centre were considered and solved using the same technique of separation of variables. The mathematical expressions obtained showed that in all cases, the deflection was expressible in terms of flexural and shear components. The maximum deflection was found to occur at the plate centre as is expected from the symmetrical nature of the problem. The shear component of the transverse deflection was found to significantly increase with significant increase in the ratio of the plate thickness to the radius $\left(h / r_{0}\right)$.
\end{abstract}

Keywords: first order shear deformable circular plate, shear deformation, flexural deformation, axisymmetrical problem, differential equations of equilibrium.

\section{Introduction}

Circular plate structures have extensive applications in aerospace, civil, structural, geotechnical, naval, and mechanical engineering [1]. They can be subjected to distributed transverse loads, in-plane loads or dynamic loads. They are classified according to the ratio of their thickness, $h$ to the diameter, $D,[2,3]$ as: thin plates $(D / h>100)$, moderately thick plates $(20<D / h<100)$, and thick plates $(D / h<3)$. Circular plates are also classified according to their material properties as: laminated plates, anisotropic plates, orthotropic plates, homogeneous plates, heterogeneous plates and isotropic plates [4-8].

The determination of displacement and deflection functions in elastic circular plates subjected to symmetrical distribution of transverse loading is a solid mechanics/theory of elasticity problem frequently encountered in the analysis and design of structural elements and systems [9]. Plate problems are generally three dimensional (3D) problems of the mathematical theory of elasticity, and the three spatial coordinates are used in their formulation. Three-dimensional plate theories have their mathematical and analytical foundations in the mathematical theory of elasticity; and the solution for 3D problems are mathematical rigorous and demanding. Only few 3D problems have so far been solved [10-14].

Theories that have been used to describe the flexural problem of circular plates include: Kirchhoff-Love plate theory also called the Classical small deformation thin plate theory (CPT) [15-18], Mindlin plate theory [19], Reissner plate theory [20-22], Von Karman plate theory [23], Shimpi's plate theory [24, 25], Levinson's plate theory [26], Reddy's plate theory [27, 28] and Modified/refined plate theories [29].

The Kirchhoff-Love plate theory is based on the Kirchhoff-Love's hypotheses which are assumptions similar to the Bernoulli-Navier's hypotheses used in the classical theory of thin (or 
slender) beams. The fundamental assumptions of the classical small deflection thin plate theory for homogeneous, isotropic elastic plates are [18]:

(i) Straight line initially normal to the middle surface to the plate remains straight and normal to the deformed middle surface of the plate and unchanged in length.

(ii) The transverse displacement $w$ is assumed to be very small. This implies that the slope of the deflection surface is small and hence the square of the slope would be considered negligible in comparison with unity.

(iii) The normal stresses in the radial and tangential (circumferential) directions $\sigma_{r r}$ and $\sigma_{\theta \theta}$ and the in-plane shear stress $\tau_{r \theta}$ are assumed to be zero at the middle surface for small deflection cases (i.e. $w \ll h)$.

(iv) The transverse normal stress $\sigma_{z z}$ is very small as compared to the other stress components and can be disregarded in the Hooke's generalised stress-strain relations without introducing significant errors i.e. $\sigma_{z z} \ll\left(\sigma_{r r}, \sigma_{\theta \theta}, \tau_{r \theta}\right)$.

(v) The middle plane remains unstrained after bending deformation and is a neutral surface (plane).

The above assumptions reduce the plate problem from a 3D mathematical theory of elasticity problem to a two dimensional (2D) approximate theory. Hence only two normal stresses $\sigma_{r r}, \sigma_{\theta \theta}$ and one in-plane transverse shear stress $\tau_{r \theta}$ are the stresses used to define the plate problem. These stresses are functions of the two coordinate variables $r$, and $\theta$.

The differential equations of equilibrium of circular plates are [6]:

$\frac{\partial M_{r r}}{\partial r}+\frac{1}{r} \frac{\partial M_{r \theta}}{\partial \theta}+\frac{M_{r r}-M_{\theta \theta}}{r}-Q_{r}=0$,

$\frac{\partial M_{r \theta}}{\partial r}+\frac{1}{r} \frac{\partial M_{\theta \theta}}{\partial \theta}+\frac{2 M_{r \theta}}{r}-Q_{\theta}=0$,

$\frac{\partial Q_{r}}{\partial r}+\frac{1}{r} \frac{\partial Q_{\theta}}{\partial \theta}+\frac{Q_{r}}{r}+q=0$,

where $M_{r r}$ are radial moments, $M_{\theta \theta}$ are circumferential moments, $M_{r \theta}$ is the twisting moment, $Q_{r}, Q_{\theta}$ are shear force in the $r$ and $\theta$ directions, $Q_{r}$ is the radial shear force, $Q_{\theta}$ is the circumferential shear force and $q$ is the transverse load distribution.

The differential equation of equilibrium is expressed in terms of the deflection of the middle surface by the fourth order biharmonic equation:

$\nabla^{4} w=\nabla^{2} \nabla^{2} w=\frac{q}{D}$

where:

$\nabla^{2}=\frac{\partial^{2}}{\partial r^{2}}+\frac{1}{r} \frac{\partial}{\partial r}+\frac{\partial^{2}}{\partial \theta^{2}}$

and $\nabla^{2}$ is the Laplacian operator in the polar coordinate system, $\nabla^{4}$ is the biharmonic operator. $D$ is the modulus of flexural rigidity of the plate.

For cases of axisymmetric or rotationally symmetric loading, the load, internal stress resultants (bending moment and shear force distributions) and the displacements become functions of one coordinate variable only, namely, $r$. In such cases, each vertical plane $(r z)$ of the plate will be a principal plane, and hence the twisting moment $M_{r \theta}$ will vanish [6]. Also, the shear force $Q_{\theta}$ and the tangential displacement $v$ will be zero. The differential equation of equilibrium of axisymmetric circular Kirchhoff-Love plate will simplify to [30, 31]: 
$\frac{d M_{r r}}{d r}+\frac{M_{r r}-M_{\theta \theta}}{r}-Q_{r}=0$,

$\frac{d Q_{r}}{d r}+\frac{Q_{r}}{r}+q=0$

The moment-curvature (moment-deflection) equations become:

$M_{r r}=-D\left(\frac{d^{2} w}{d r^{2}}+\frac{\mu}{r} \frac{d w}{d r}\right)$,
$M_{\theta \theta}=-D\left(\mu \frac{d^{2} w}{d r^{2}}+\frac{1}{r} \frac{d w}{d r}\right)$.

The radial shear force $Q_{r}$ is:

$Q_{r}=-D \frac{d}{d r} \nabla^{2} w$

$D$ is related to the Young's modulus, $E$ and the Poisson's ratio, $\mu$ by:

$D=\frac{E h^{3}}{12\left(1-\mu^{2}\right)}$,

where $h$ is the plate thickness.

$\nabla^{2}$ is the Laplacian in axisymmetric polar coordinates:

$\nabla^{2}=\frac{d^{2}}{d r^{2}}+\frac{1}{r} \frac{d}{d r}$

Kirchhoff's theory, through satisfactory for thin plates, has been found to be unsatisfactory as the plate thickness increases. Its major limitation is the disregard for shear deformation which renders it incapable of accurately describing the behaviour of moderately thick plates; where shear deformation will be significant. Von Karman's theory for the large deflection, axisymmetric bending of a circular plate which is either simply supported or clamped at the boundary can be represented by the system of coupled non-linear partial differential equations [23] expressed in terms of two unknowns $w(r)$ and $\phi(r)$ :

$\frac{E h^{3}}{12\left(1-\mu^{2}\right)} \nabla^{2} w-\frac{1}{r} \frac{d}{d r}\left(\frac{d \phi}{d r} \frac{d w}{d r}\right)=\frac{p(r)}{h}$,

$\frac{1}{E} \nabla^{2} \phi+\frac{1}{r} \frac{d w}{d r} \frac{d^{2} w}{d r^{2}}=0$,

where:

$\nabla^{2}=\frac{1}{r} \frac{d}{d r} r \frac{d}{d r}$

and $E$ and $\mu$ are the Young's modulus and Poisson's ratio, respectively. $h$ is the plate thickness, $r$ is the radial coordinate variable. $\phi$ is the Airy stress function, $p(r)$ is the applied radially symmetric load distribution.

The study is focused on first order shear deformable circular plate theory of Mindlin. The theory is capable of describing the behaviours of moderately thick plates where shear deformations will contribute to the flexural behaviour. 


\subsection{Review of solution methods for the boundary value problem of circular plates}

The boundary value problem (BVP) of circular plates is represented generally by differential equations and boundary conditions. They are solved using techniques for solving ordinary and partial differential equations. The problems can also be formulated in weighted residual form or using variational calculus. Thus, the methods used in the literature for the solution of the plate problem include: Separation of variables, Integral transforms, Eigen function expansions, Fourier series, etc. The numerical methods used in the literature include: Galerkin method, Kantorovich method, Ritz method, Finite Element method and boundary element methods [32-35].

\subsection{Justification for the Mindlin First order shear deformable theory}

The classical two dimensional (2D) Kirchhoff - theory of flexure of elastic circular (thin) plates disregards the axial shear strains and normal stress. Hence the Kirchhoff circular plate theory cannot be expected to give good estimates of the deflection and internal forces in circular plates with a thickness to radius ratio exceeding 0.05. Mindlin [19] considered the effect of rotatory inertia and shear deformation on the flexural behaviour of isotropic circular plates and derived from first principles the governing equations for a more comprehensive 2D theory called the Mindlin first order shear deformation plate theory FSDT for circular plates.

Mindlin's theory is analogous to the Timoshenko's theory for beams, which takes into consideration shear deformation in the flexural analysis of beams. Mindlin's FSDT for circular plates satisfies the stress-strain equations for transverse shear stresses and shear strains by the use of shear modification (correction) factors $k_{s}$. The shear modification factors depend upon the plate material, geometry, cross-sectional shape, loading, and support/boundary conditions.

However, a fundamental inadequacy of the Mindlin's FSDT is that all the equations of three dimensional theory of elasticity are not satisfied by the theory, and only deflections of the middle surfaces (planes)of the plates are considered [36]. Despite the obvious limitation, the Mindlin 2D plate theory plays a significant role in the validation of results for deflections and internal stress resultants of thick plate theories [36].

Reddy's higher order shear deformation plate theory (HSDPT) assumes a quadratic (parabolic) variation of transverse shear stress through the plate thickness such that shear stress free boundary conditions required for the top and bottom surfaces of the plate are automatically satisfied. Hence the Reddy theory does not require shear correction factors.

\subsection{Advantages of mathematical solutions over numerical solutions in plate bending analysis}

The focus of the present study is to derive mathematical solutions, also called closed form or analytical solutions to the flexural problem of Mindlin circular plates; due to the obvious merits of the mathematical solutions over the numerical or approximate solutions. The advantages of the mathematical solutions are:

(i) The mathematical solutions aim to derive using rigorous mathematical techniques, the "exact" solutions to the governing partial differential equations of the Mindlin circular plate theory, which are valid at all points on the circular plate domain as well as on the boundaries; for given loading and restraint conditions. The solutions derived in a mathematical approach are called 'exact' within the limitations of the foundational theory used in formulating the plate problem, in this case, the Mindlin first order shear deformation theory. However, the numerical solutions aim to obtain approximate solutions to the plate flexure problem which may not be valid solutions at all points on the solution domain; nor at the boundaries.

(ii) The derivation of analytical solutions is important since they serve as benchmark solutions for comparing the accuracy of numerical solutions.

(iii) The advantages of mathematical solutions include its better accuracy as compared with 
numerical solutions and broad generality in solving plate flexure problems with given loads and restraint conditions.

(iv) The mathematical derivation of the solutions provide a systematic framework for solving the flexural problems of Mindlin circular plates with different edge restraint conditions.

Despite the advantages of mathematical solutions, exact analytical solutions for plate bending problems are very limited, even for the classical Kirchhoff-thin plate theory. They are usually mathematically rigorous, and mathematical solutions do not exist for many plate bending problems involving complicated restraint conditions and complex load distributions. For plates of arbitrary shape, the solution of the governing plate equations, even in the classical Kirchhoff thin plate theory is a non-trivial matter and in general, an exact analytical/mathematical solution does not exist [37].

Even for the classical Kirchhoff plate theory, the mathematical rigours of obtaining closed form solutions to the BVP are so complex that mathematical solutions leading to formula for stresses or deflections are only possible/available for a few simple cases of geometry, boundary conditions and loading [38].

\subsection{Research aim and objectives}

The general aim of this paper is to solve and obtain mathematical solutions for the boundary value problem of first order shear deformable circular plates. The general objectives are:

(i) to solve the governing boundary value problem of flexure of first order shear deformable circular plates under arbitrary distributed load $p(r)$ and obtain general solutions for the generalised displacements in terms of constants of integration.

(ii) to find mathematical solutions to the flexural problem of first order shear deformable circular plates with clamped edge $\left(r=r_{0}\right)$ for the case of (a) uniformly distributed transverse load over the entire plate domain, and (b) point load $P_{0}$ applied at the centre $(r=0)$ of the plate.

(iii) to find analytical solutions for the flexural problem of first order shear deformable circular plates with simply supported edges for the case of uniformly distributed transverse load over the entire plate domain.

\section{Theoretical framework}

Axisymmetric problems of the theory of elasticity are governed by the requirements of the strain displacement relations, the differential equations of equilibrium and the stress-strain law. The differential equations of equilibrium are given by:

$$
\begin{aligned}
& \frac{\partial \sigma_{r r}}{\partial r}+\frac{1}{r} \frac{\partial \tau_{r \theta}}{\partial \theta}+\frac{1}{r}\left(\sigma_{r r}-\sigma_{\theta \theta}\right)=0, \\
& \frac{\partial \tau_{r \theta}}{\partial r}+\frac{1}{r} \frac{\partial \tau_{\theta \theta}}{\partial \theta}+\frac{2 \tau_{r \theta}}{r}=0,
\end{aligned}
$$

where $\sigma_{r r}$ and $\sigma_{\theta \theta}$ are the radial and circumferential stresses and $\tau_{r \theta}$ is the shear stress.

The strain-displacement relations for axisymmetric small displacement problems are given by the three equations:

$$
\begin{aligned}
& \varepsilon_{r r}=\frac{\partial u_{r}}{\partial r}, \\
& \varepsilon_{\theta \theta}=\frac{1}{r} \frac{\partial u_{\theta}}{\partial \theta}+\frac{u_{r}}{r}, \\
& 2 \varepsilon_{r \theta}=\gamma_{r \theta}=\frac{1}{r} \frac{\partial u_{r}}{\partial \theta}+\frac{\partial u_{\theta}}{\partial r}-\frac{u_{\theta}}{r},
\end{aligned}
$$

where $\varepsilon_{r r}$ is the radial strain (normal strain in the radial direction), $\varepsilon_{\theta \theta}$ is the circumferential 
normal strain, $\varepsilon_{r \theta}$ is the shear strain in the $r \theta$ direction, $u_{r}$ is the radial component of displacement, $u_{\theta}$ is the circumferential component (tangential component) of the displacement.

The strain-stress relations in the axisymmetric (polar) coordinates are for plane stress conditions:

$$
\begin{aligned}
& \varepsilon_{r r}=\frac{1}{E}\left(\sigma_{r r}-\mu \sigma_{\theta \theta}\right), \\
& \varepsilon_{\theta \theta}=\frac{1}{E}\left(\sigma_{\theta \theta}-\mu \sigma_{r r}\right), \\
& \varepsilon_{z z}=-\frac{\mu}{E}\left(\sigma_{r r}+\sigma_{\theta \theta}\right), \\
& 2 \varepsilon_{r \theta}=\frac{2(1+\mu)}{E} \tau_{r \theta}=\frac{\tau_{r \theta}}{G}=\gamma_{r \theta},
\end{aligned}
$$

and for plane strain conditions:

$$
\begin{aligned}
& \varepsilon_{r r}=\frac{1+\mu}{E}\left((1-\mu) \sigma_{r r}-\mu \sigma_{\theta \theta}\right), \\
& \varepsilon_{\theta \theta}=\frac{1+\mu}{E}\left(\mu \sigma_{r r}+(1-\mu) \sigma_{\theta \theta}\right), \\
& \gamma_{r \theta}=\frac{\tau_{r \theta}}{G}=\frac{2(1+\mu) \tau_{r \theta}}{E},
\end{aligned}
$$

where $E$ is the Young's modulus of elasticity, $\mu$ is the Poisson's ratio, and $G$ is the shear modulus. $G$ is related to the Young's modulus $E$ by:

$$
G=\frac{E}{2(1+\mu)} .
$$

\section{Governing equations}

The differential equations of equilibrium of first order shear deformable circular plates are given by the system of three partial differential equations in terms of the stress resultants, $M_{r r}$, $M_{\theta \theta}, M_{r \theta}, Q_{r}$ and $Q_{\theta}$ as follows:

$$
\begin{aligned}
& -\frac{1}{r}\left(\frac{\partial}{\partial r}\left(r M_{r r}\right)+\frac{\partial M_{r \theta}}{\partial \theta}-M_{\theta \theta}\right)+Q_{r}=0 \\
& -\frac{1}{r}\left(\frac{\partial}{\partial r}\left(r M_{r \theta}\right)+\frac{\partial M_{\theta \theta}}{\partial \theta}+M_{r \theta}\right)+Q_{\theta}=0 \\
& -\frac{1}{r}\left(\frac{\partial}{\partial r}\left(r Q_{r}\right)+\frac{\partial \theta_{\theta}}{\partial \theta}\right)-p=0 .
\end{aligned}
$$

The stress resultants for first order shear deformable circular plates are expressed in terms of the displacements $w_{0}, \varphi_{r}$ and $\varphi_{\theta}$ as:

$$
\begin{aligned}
& M_{r r}=D\left(\frac{\partial \varphi_{r}}{\partial r}+\frac{\mu}{r}\left(\varphi_{r}+\frac{1}{r} \frac{\partial \varphi_{\theta}}{\partial \theta}\right)\right), \\
& M_{\theta \theta}=D\left(\mu \frac{\partial \varphi_{r}}{\partial r}+\frac{1}{r}\left(\varphi_{r}+\frac{1}{r} \frac{\partial \varphi_{\theta}}{\partial \theta}\right)\right), \\
& M_{r \theta}=\frac{D(1-\mu)}{r}\left(\frac{\partial \varphi_{r}}{\partial \theta}+r \frac{\partial \varphi_{\theta}}{\partial r}-\varphi_{\theta}\right),
\end{aligned}
$$


$Q_{r}=k_{s} \int_{-h / 2}^{h / 2} \tau_{r z} d z=k_{s} G h\left(\varphi_{r}+\frac{\partial w_{0}}{\partial r}\right)$,
$Q_{\theta}=k_{s} \int_{-h / 2}^{h / 2} \tau_{\theta z} d z=k_{s} G h\left(\varphi_{\theta}+\frac{1}{r} \frac{\partial w_{0}}{\partial \theta}\right)$,

where $k_{s}$ is the shear correction factor.

For axisymmetric problems:

$\varphi_{\theta}=0$,

$M_{r \theta}=0$,

$Q_{\theta}=0$.

Consequently, the differential equations of equilibrium of axially symmetric problems simplify to the system of two partial differential equations:

$$
\begin{aligned}
& -\frac{1}{r}\left(\frac{\partial}{\partial r}\left(r M_{r r}\right)-M_{\theta \theta}\right)+Q_{r}=0, \\
& -\frac{1}{r} \frac{d}{d r}\left(r Q_{r}\right)-p(r)=0 .
\end{aligned}
$$

For axially symmetric problems of first order shear deformable circular plates, the internal stress resultants (bending moments $M_{r r}, M_{\theta \theta}$ and shear force distributions $Q_{r}$ ) are related to the generalized displacement functions $\varphi_{r}, w_{0}$ as follows:

$$
\begin{aligned}
& M_{r r}=D\left(\frac{d \varphi_{r}}{d r}+\mu \frac{\varphi_{r}}{r}\right), \\
& M_{\theta \theta}=D\left(\mu \frac{d \varphi_{r}}{d r}+\frac{\varphi_{r}}{r}\right), \\
& Q_{r}=k_{s} G h\left(\varphi_{r}+\frac{d w_{0}}{d r}\right) .
\end{aligned}
$$

\section{General solution of the boundary value problem of first order shear deformable circular plates under transverse flexure}

The differential equation of equilibrium Eq. (41) is observed to be variable separable. We thus seek a solution using the method of separation of variables. Eq. (41) is rewritten as:

$-\frac{1}{r} \frac{d}{d r}\left(r Q_{r}\right)=p(r)$

Separation of variables, and integration yields:

$\int d\left(r Q_{r}\right)=r Q_{r}=-\int r p(r) d r+c_{0}$

where $c_{0}$ is a constant of integration.

From Eq. (40), we have:

$\frac{\partial}{\partial r}\left(r M_{r r}\right)-M_{\theta \theta}=r \frac{d M_{r r}}{d r}+M_{r r}-M_{\theta \theta}=r Q_{r}$

Substitution of Eqs. (42) and (43) into Eq. (47) yields after simplification: 
$D\left(r \frac{d^{2} \varphi_{r}}{d r^{2}}+\frac{d \varphi_{r}}{d r}-\frac{\varphi_{r}}{r}\right)=r Q_{r}=D\left(r \frac{d}{d r}\left(\frac{d \varphi_{r}}{d r}+\frac{\varphi_{r}}{r}\right)\right)=r Q_{r}=D r\left(\frac{1}{r} \frac{d}{d r}\left(r \varphi_{r}\right)\right)$.

Using Eq. (46) we have:

$\operatorname{Dr} \frac{d}{d r}\left(\frac{d \varphi_{r}}{d r}+\frac{\varphi_{r}}{r}\right)=-\int r p(r) d r+c_{0}$

Solution by successive integration yields:

$D \varphi_{r}=-\frac{1}{r} \int r \int \frac{1}{r} \int r p(r) d r d r d r+\frac{c_{0} r}{4}(2 \log r-1)+\frac{r c_{1}}{2}+\frac{c_{2}}{r}$,

where $c_{1}$ and $c_{2}$ are constants of integration.

Thus, we obtain:

$D \varphi_{r}=-I^{\prime}(r)+\frac{r}{4}(2 \log r-1) c_{0}+\frac{r}{2} c_{1}+\frac{c_{2}}{r}$,

where:

$I^{\prime}(r)=\frac{1}{r} \int r \int \frac{1}{r} \int r p(r) d r d r d r$,

$I_{r}(r)=\int \frac{1}{r} \int r \int \frac{1}{r} \int r p(r) d r d r d r d r$.

The solution for $w_{0}$ is obtained using Eqs. (44) and (46).

Thus:

$r Q_{r}=r k_{s} G h\left(\varphi_{r}+\frac{d w_{0}}{d r}\right)=-\int r p(r) d r+c_{0}$,

$k_{s} G h \frac{d w_{0}}{d r}=-\frac{1}{r} \int r p(r) d r+\frac{c_{0}}{r}-k_{s} G h \varphi_{r}$,

$k_{s} G h \frac{d w_{0}}{d r}=-\frac{1}{r} \int r p(r) d r+\frac{c_{0}}{r}-\frac{k_{s} G h}{D}\left(-I^{\prime}(r)+\frac{c_{0} r}{4}(2 \log r-1)+\frac{r c_{1}}{2}+\frac{c_{2}}{r}\right)$.

Integrating both sides of Eq. (56) with respect to $r$, we obtain:

$$
\begin{aligned}
& k_{s} G h w_{0}(r)=-\int \frac{1}{r} \int r p(r) d r d r \\
& \quad+\int \frac{c_{0}}{r} d r-\frac{k_{s} G h}{D} \int\left(-I^{\prime}(r)+\frac{c_{0} r}{4}(2 \log r-1)+\frac{c_{1} r}{2}+\frac{c_{2}}{r}\right) d r .
\end{aligned}
$$

Thus:

$$
\begin{aligned}
& k_{s} G h w_{0}(r)=-\int \frac{1}{r} \int r p(r) d r d r+c_{0} \log r \\
& -\frac{k_{s} G h}{D}\left(-I(r)+\frac{c_{0} r^{2}}{4}(\log r-1)+\frac{r^{2} c_{1}}{4}+c_{2} \log r\right)+c_{3},
\end{aligned}
$$


where $c_{3}$ is a fourth constant of integration.

The equations obtained for $\varphi_{0}(r)$ and $w_{0}(r)$ given respectively by Eqs. (51) and (58) are the general solutions for the boundary value problem of first order shear deformable circular plates subject to the transverse distributed load $p(r)$. The general solution is obtained in terms of four unknown constants of integration $c_{0}, c_{1}, c_{2}$ and $c_{3}$. These four constants of integration can be determined from the essential and natural boundary conditions for particular problems of first order shear deformable circular plates.

This paper considers three particular problems/cases, namely: (i) solid first order shear deformable circular plate with clamped edges and under uniformly distributed transverse load (ii) solid first order shear deformable circular plate with clamped edges and under point load $P_{0}$ applied at the centre (iii) simply supported solid shear deformable circular plate under uniformly distributed load $p_{0}$.

\subsection{Case 1: First order shear deformable solid circular plate with clamped edge and under uniform transverse load distribution}

For bounded solutions for the rotation $\varphi_{r}, \varphi_{r}$ is required to be non-singular at $r=0$, hence:

$c_{2}=0$.

For solid circular plates that are not subject to point load at the centre, $r=0$, the shear force at the centre is required to vanish. This yields from Eq. (46):

$c_{0}=0$.

For clamped solid circular first order shear deformable plates of radius $r_{0}$, the boundary conditions of the clamped edge $r=r_{0}$ are:

$w_{0}\left(r=r_{0}\right)=0$,

$\varphi_{r}\left(r=r_{0}\right)=0$.

For uniformly distributed transverse load of intensity $p_{0}, p(r)=p_{0}$ :

$I(r)=\int \frac{1}{r} \int_{0} r \int \frac{1}{r} \int r p_{0} d r d r d r d r=p_{0} \int \frac{1}{r} \int r \int \frac{1}{r} \int r d r d r d r d r=\frac{p_{0} r^{4}}{64}$,
$I^{\prime}(r)=\frac{p_{0} r^{3}}{16}$.

The solution for $w_{0}(r)$ becomes:

$$
\begin{aligned}
& k_{s} G h w_{0}(r)=-\int \frac{1}{r} \int r p_{0} d r d r-\frac{k_{s} G h}{D}\left(\frac{p_{0} r^{4}}{64}+\frac{r^{2} c_{1}}{4}\right)+c_{3}, \\
& k_{s} G h w_{0}(r)=\frac{-p_{0} r^{2}}{4}-\frac{k_{s} G h}{D}\left(\frac{p_{0} r^{4}}{64}+\frac{r^{2} c_{1}}{4}\right)+c_{3} .
\end{aligned}
$$

Similarly, the solution for $\varphi_{r}$ becomes:

$D \varphi_{r}=\frac{-p_{0} r^{3}}{16}+\frac{r c_{1}}{2}$

Using the boundary condition Eq. (62) in Eq. (67) we obtain: 
$c_{1}=\frac{p_{0} r_{0}^{2}}{8}$

Application of the boundary condition Eq. (61) in Eq. (66) yields:

$c_{3}=\frac{p_{0} r_{0}^{2}}{4}+\frac{k_{s} G h}{D} \frac{p_{0} r_{0}^{4}}{64}$

Substitution of $c_{1}$ and $c_{3}$ into the equation for $w_{0}(r)$ and $\varphi_{r}$ give the solutions for this case as:

$$
\begin{aligned}
& w_{0}(r)=\frac{p_{0} r_{0}^{4}}{64 D}\left(1-\left(\frac{r}{r_{0}}\right)^{2}\right)^{2}+\frac{p_{0} r_{0}^{2}}{4 k_{s} G h}\left(1-\left(\frac{r}{r_{0}}\right)^{2}\right) \\
& =\frac{p_{0} r_{0}^{4}}{64 D}\left(1-2\left(\frac{r}{r_{0}}\right)^{2}+\left(\frac{r}{r_{0}}\right)^{4}\right)+\frac{p_{0} r_{0}^{2}}{4 k_{s} G h}\left(1-\left(\frac{r}{r_{0}}\right)^{2}\right) \\
& \varphi_{r}(r)=\frac{p_{0} r_{0}^{2} r}{16 D}\left(1-\left(\frac{r}{r_{0}}\right)^{2}\right), \\
& \varphi_{r}(r)=\frac{p_{0} r_{0}^{3}}{16 D}\left(\frac{r}{r_{0}}\right)\left(1-\left(\frac{r}{r_{0}}\right)^{2}\right)=\frac{p_{0} r_{0}^{3}}{16 D}\left(\frac{r}{r_{0}}-\left(\frac{r}{r_{0}}\right)^{3}\right) .
\end{aligned}
$$

The transverse deflection $w_{0}(r)$ can be decomposed into two components, a flexural (bending) component $w_{0}^{f}(r)$ and a shear component $w_{0}^{s}(r)$ as follows:

$w(r)=w_{0}^{f}(r)+w_{0}^{s}(r)$,

where:

$$
\begin{aligned}
& w_{0}^{f}(r)=\frac{p_{0} r_{0}^{4}}{64 D}\left(1-\left(\frac{r}{r_{0}}\right)^{2}\right)^{2}, \\
& w_{0}^{s}(r)=\frac{p_{0} r_{0}^{2}}{4 k_{s} G h}\left(1-\left(\frac{r}{r_{0}}\right)^{2}\right) .
\end{aligned}
$$

\subsubsection{Maximum deflection}

The maximum deflection $w_{\max }$ is found to occur at the centre of the plate where $r=0$, and is given by:

$$
\begin{aligned}
& w_{\max }=\frac{p_{0} r_{0}^{4}}{64 D}+\frac{p_{0} r_{0}^{2}}{4 k_{s} G h^{\prime}} \\
& w_{\max }=\frac{p_{0} r_{0}^{4}}{64 D}\left(1+\frac{8}{3 k_{s}(1-\mu)}\left(\frac{h}{r_{0}}\right)^{2}\right) .
\end{aligned}
$$

For $k_{s}=5 / 6$, and $\mu=0.30$ :

$w_{\max }=\frac{p_{0} r_{0}^{4}}{64 D}\left(1+\frac{8}{1.75}\left(\frac{h}{r_{0}}\right)^{2}\right)$.

Table 1 shows the maximum deflection of first order shear deformable solid circular plates with clamped edge for the case of uniformly distributed load computed for varying values of $h / r_{0}$.

Table 2 shows the difference between $w_{\max }$ the maximum deflection obtained by the first 
order shear deformable circular plate theory and the classical Kirchhoff-Love circular plate theory for various values of $h / r_{0}$ for the case of clamped edge and uniformly distributed load.

Table 1. Maximum deflection of first order shear deformable circular plates with clamped edges and under uniformly distributed load $p_{0}$

\begin{tabular}{|c|c|}
\hline$\frac{h}{r_{0}}$ & $\mathrm{w}_{\max }\left(\times \frac{p_{0} r_{0}^{4}}{D}\right)$ \\
\hline 0.005 & $1.562679 \times 10^{-2}$ \\
\hline 0.01 & $1.563214 \times 10^{-2}$ \\
\hline 0.05 & $1.580357 \times 10^{-2}$ \\
\hline 0.10 & $1.633929 \times 10^{-2}$ \\
\hline 0.2 & $1.848214 \times 10^{-2}$ \\
\hline 0.3 & $2.220536 \times 10^{-2}$ \\
\hline
\end{tabular}

Table 2. Difference between the maximum deflections of first order shear deformable circular plates and the Kirchhoff-Love circular plate with clamped edges (case of uniformly distributed load)

\begin{tabular}{|c|c|c|}
\hline$h / r_{0}$ & $\left(w_{\max }^{F S D P}-w_{\max }^{C P T}\right)$ & Difference $\%$ \\
\hline 0.005 & $1.142857 \times 10^{-4}$ & 0.0114 \\
\hline 0.01 & $4.57142 \times 10^{-4}$ & 0.0457 \\
\hline 0.05 & $1.142857 \times 10^{-2}$ & 1.1429 \\
\hline 0.1 & $4.57142 \times 10^{-2}$ & 4.5714 \\
\hline 0.2 & $18.2857 \times 10^{-2}$ & 18.2857 \\
\hline 0.3 & $41.142857 \times 10^{-2}$ & 41.1429 \\
\hline
\end{tabular}

The bending moment and shear force distributions are found by substitution of Eq. (72) into Eqs. (42) and (43) as:

$$
\begin{aligned}
& M_{r r}(r)=\frac{p_{0} r_{0}^{2}}{16}\left[(1+\mu)-(3+\mu)\left(\frac{r}{r_{0}}\right)^{2}\right], \\
& M_{\theta \theta}(r)=\frac{p_{0} r_{0}^{2}}{16}\left[(1+\mu)-(1+3 \mu)\left(\frac{r}{r_{0}}\right)^{2}\right] .
\end{aligned}
$$

The bending stresses are found as:

$$
\begin{aligned}
& \sigma_{r r}(r, z)=\frac{3 p_{0} r_{0}^{2} z}{4 h^{3}}\left[(1+\mu)-(3+\mu)\left(\frac{r}{r_{0}}\right)^{2}\right] . \\
& \sigma_{\theta \theta}(r, z)=\frac{3 p_{0} r_{0}^{2} z}{4 h^{3}}\left[(1+\mu)-(1+3 \mu)\left(\frac{r}{r_{0}}\right)^{2}\right] .
\end{aligned}
$$

The shear stress distribution $\tau_{r z}$ is given by:

$$
\begin{gathered}
\tau_{r z}(r)=G \gamma_{r z}=G\left(\varphi_{r}+\frac{d w_{0}(r)}{d r}\right) \\
=G\left(\frac{p_{0} r_{0}^{4}}{64 D}\left(\frac{-4 r}{r_{0}^{2}}+\frac{4 r^{3}}{r_{0}^{4}}\right)+\frac{p_{0} r_{0}^{2}}{4 k_{s} G h}\left(-\frac{2 r}{r_{0}^{2}}\right)+\frac{p_{0} r_{0}^{3}}{16 D}\left(\frac{r}{r_{0}}-\left(\frac{r}{r_{0}}\right)^{3}\right)\right) \\
=G\left(\frac{p_{0} r_{0}^{3}}{16 D}\left(\frac{-r}{r_{0}}+\left(\frac{r}{r_{0}}\right)^{3}+\left(\frac{r}{r_{0}}\right)-\left(\frac{r}{r_{0}}\right)^{3}\right)-\frac{p_{0} r}{2 k_{s} G h}\right)=-\frac{p_{0} r}{2 k_{s} h} .
\end{gathered}
$$

The shear force distribution $Q_{r}(r)$ is from Eq. (75): 
$Q_{r}(r)=k_{s} G h \frac{d w_{0}^{S}}{d r}=k_{s} G h \frac{p_{0} r_{0}^{2}}{4 k_{s} G h}\left(\frac{-2 r}{r_{0}^{2}}\right)=-\frac{p_{0} r}{2}=k_{s} h \tau_{r z}=k \int_{-h / 2}^{h / 2} \tau_{r z} d z$.

The deflection and rotation functions satisfy the boundary conditions at the clamped edge since upon substitution of $r=r_{0}$ into the equations, Eqs. (61) and (62) are satisfied. They also satisfy the conditions of symmetry since $\varphi_{r}(r=0)=0$.

\subsection{Case 2: First order shear deformable solid circular plate with clamped edge and point load $P_{0}$ applied at the centre, $r=0$}

For first order shear deformable solid circular plate (FSDP) subject to a point load at the centre, enforcement of boundary conditions yield:

$c_{0}=-\frac{P_{0}}{2 \pi}$

The requirement of boundedness of $w_{0}(r)$ also require that:

$c_{2}=0$.

Then, the displacements become:

$$
\begin{aligned}
& D \varphi_{r}=\frac{r}{4}(2 \log r-1)\left(-\frac{P_{0}}{2 \pi}\right)+\frac{r c_{1}}{2}, \\
& k_{S} G h w_{0}(r)=-\int \frac{1}{r} \int r p(r) d r d r-\frac{P_{0}}{2 \pi} \log r \\
& \quad-\frac{k_{s} G h}{D}\left(-I(r)-\frac{P_{0}}{2 \pi} \frac{r^{2}}{4}(\log r-1)+\frac{r^{2}}{4} \frac{P_{0}}{4 \pi}\left(2 \log r_{0}-1\right)\right)+c_{3} .
\end{aligned}
$$

Enforcement of the boundary conditions Eqs. (61) and (62) in Eqs. (87) and (88) yield the following constants of integration:

$$
\begin{aligned}
c_{1} & =\frac{P_{0}}{2 \pi}\left(2 \log r_{0}-1\right), \\
c_{3}= & \frac{P_{0} \log r_{0}}{2 \pi}+\frac{k_{s} G h}{D} \frac{P_{0} r_{0}^{2}}{16 \pi} .
\end{aligned}
$$

Hence the solutions for transverse deflections and rotation functions become:

$$
\begin{aligned}
& w_{0}(r)=\frac{P_{0} r_{0}^{2}}{16 \pi D}\left(1-\left(\frac{r}{r_{0}}\right)^{2}+2\left(\frac{r}{r_{0}}\right)^{2} \log _{e}\left(\frac{r}{r_{0}}\right)\right)-\frac{P_{0}}{2 \pi k_{s} G h} \log _{e}\left(\frac{r}{r_{0}}\right)=w_{0}^{f}(r)+w_{0}^{s}(r), \\
& \varphi(r)=\frac{-P_{0} r}{4 \pi D} \log _{e}\left(\frac{r}{r_{0}}\right) \\
& w_{0}(r)=\frac{P_{0} r_{0}^{2}}{16 \pi D}\left(1-\left(\frac{r}{r_{0}}\right)^{2}+2\left(\frac{r}{r_{0}}\right)^{2} \log _{e}\left(\frac{r}{r_{0}}\right)\right)-\frac{P_{0} h^{3}}{2 \pi k_{s} h 6 D(1-\mu)} \log _{e}\left(\frac{r}{r_{0}}\right) \\
& =\frac{P_{0} r_{0}^{2}}{16 \pi D}\left(1-\left(\frac{r}{r_{0}}\right)^{2}+2\left(\frac{r}{r_{0}}\right)^{2} \log _{e}\left(\frac{r}{r_{0}}\right)\right)-\frac{P_{0} h^{2}}{12 \pi D k_{s}(1-\mu)} \log _{e}\left(\frac{r}{r_{0}}\right) .
\end{aligned}
$$




\subsection{Case 3: First order shear deformable solid circular plate with simply supported edge and under uniformly distributed transverse load}

The essential and natural boundary conditions of the simple supports at the edge $r=r_{0}$ are:

$w\left(r=r_{0}\right)=0$,

$M_{r r}\left(r=r_{0}\right)=0$.

At the centre, $r=0$ :

$\left.\left(r Q_{r}\right)\right|_{r=0}=0$.

From Eq. (93) it is found that:

$c_{0}=0$.

For non singular solutions for the displacements, the expression for $\varphi_{r}$ gives the condition for bounded solutions as:

$c_{2}=0$.

Hence, for simply supported FSDP under uniformly distributed transverse load:

$k_{S} G h w_{0}(r)=-\int \frac{1}{r} \int r p(r) d r d r-\frac{k_{s} G h}{D}\left(-I(r)+\frac{r^{2} c_{1}}{4}\right)+c_{3}$,

$D \varphi_{r}=-I^{\prime}(r)+\left(\frac{r}{2} \log r-\frac{r}{4}\right) c_{0}+\frac{r}{2} c_{1}+\frac{c_{2}}{r}$,

$D \varphi_{r}=-I^{\prime}(r)+\frac{r}{2} c_{1}$,

$D \varphi^{\prime}{ }_{r}=-I^{\prime \prime}(r)+\frac{c_{1}}{2}$

$\varphi^{\prime}{ }_{r}=\frac{1}{D}\left(-I^{\prime \prime}(r)+\frac{c_{1}}{2}\right)$,

$M_{r r}=D\left(\frac{\mu}{r D}\left(-I^{\prime}(r)+\frac{r}{2} c_{1}\right)+\frac{1}{D}\left(\frac{c_{1}}{2}-I^{\prime \prime}(r)\right)\right)=\frac{\mu}{r}\left(\frac{r c_{1}}{2}-I^{\prime}(r)\right)+\frac{c_{1}}{2}-I^{\prime \prime}(r)$,

$M_{r r}(r)=\frac{\mu}{r}\left(r \frac{c_{1}}{2}-\frac{p r^{3}}{16}\right)+\frac{c_{1}}{2}-\frac{3 p_{0} r^{2}}{16}$.

The force boundary condition yields:

$M_{r r}\left(r=r_{0}\right)=\frac{\mu}{r_{0}}\left(\frac{r_{0} c_{1}}{2}-\frac{p_{0} r_{0}^{3}}{16}\right)+\frac{c_{1}}{2}-\frac{3 p_{0} r_{0}^{2}}{16}=0$.

Solving:

$c_{1}=\frac{p_{0} r_{0}^{2}}{8}\left(\frac{3+\mu}{1+\mu}\right)$.

The deflection boundary condition at the simply supported edge gives:

$w\left(r=r_{0}\right)=-\frac{p_{0} r_{0}^{2}}{4}+\frac{p_{0} r_{0}^{4} k_{s} G h}{64 D}-\frac{k_{s} G h r_{0}^{2}}{4 D} \frac{p_{0} r_{0}^{2}}{8}\left(\frac{3+\mu}{1+\mu}\right)+c_{3}=0$. 
Solving:

$c_{3}=\frac{k_{s} G h}{D} \frac{p_{0} r_{0}^{4}}{64}\left(\frac{5+\mu}{1+\mu}\right)+\frac{p_{0} r_{0}^{2}}{4}$.

Thus, the solutions for the displacements become:

$$
\begin{aligned}
& w_{0}(r)=\frac{p_{0} r_{0}^{4}}{64 D}\left(\left(\frac{5+\mu}{1+\mu}\right)-\left(\frac{r}{r_{0}}\right)^{4}-2\left(\frac{r}{r_{0}}\right)^{2}\left(\frac{3+\mu}{1+\mu}\right)\right)+\frac{p_{0} r_{0}^{2}}{4 k_{s} G h}\left(1-\left(\frac{r}{r_{0}}\right)^{2}\right) \\
& =w_{0}^{f}(r)+w_{0}^{s}(r), \\
& \varphi(r)=\frac{p_{0} r_{0}^{3}}{16 D}\left(\frac{r}{r_{0}}\right)\left(\frac{3+\mu}{1+\mu}-\left(\frac{r}{r_{0}}\right)^{2}\right) .
\end{aligned}
$$

\subsubsection{Maximum deflection}

The maximum deflection $w_{\max }$ occurs at the plate centre, $r=0$, and is found as:

$w_{\max }=w(r=0)=\frac{p_{0} r_{0}^{4}}{64 D}\left(\frac{5+\mu}{1+\mu}\right)+\frac{p_{0} r_{0}^{2}}{4 k_{s} G h^{\prime}}$
$w_{\max }=\frac{p_{0} r_{0}^{4}}{64 D}\left(\frac{5+\mu}{1+\mu}\right)+\frac{p_{0} r_{0}^{2}}{4 k_{s} h G}$,
$w_{\max }=\frac{p_{0} r_{0}^{4}}{D}\left(\left(\frac{5+\mu}{1+\mu}\right) \frac{1}{64}+\frac{1}{24(1-\mu) k_{s}}\left(\frac{h}{r_{0}}\right)^{2}\right)$.

For $\mu=0.3, k_{s}=5 / 6$ :

$w_{\max }=\frac{p_{0} r_{0}^{4}}{D}\left(\left(\frac{5.3}{1.3}\right) \frac{1}{64}+\frac{1}{24(0.7) \frac{5}{6}}\left(\frac{h}{r_{0}}\right)^{2}\right)$.

\subsubsection{Maximum rotation}

The maximum rotation $\left(\varphi_{\max }\right)$ occurs at the edge $r=r_{0}$ is given by:

$\varphi_{\max }=\varphi_{r}\left(r=r_{0}\right)=\frac{p_{0} r_{0}^{3}}{8 D(1+\mu)}$.

\subsubsection{Bending moments}

The bending moment distribution $M_{r r}(r)$ is given by:

$$
\begin{aligned}
& M_{r r}=\mu\left(\frac{c_{1}}{2}-\frac{p_{0} r^{2}}{16}\right)+\frac{c_{1}}{2}-\frac{3 p_{0} r^{2}}{16}=\frac{\mu c_{1}}{2}-\frac{\mu p_{0} r^{2}}{16}+\frac{c_{1}}{2}-\frac{3 p_{0} r^{2}}{16} \\
& M_{r r}(r)=\frac{c_{1}}{2}(1+\mu)-\frac{p_{0} r^{2}}{16}(3+\mu)=\frac{p_{0} r_{0}^{2}}{16}(3+\mu)-\frac{p_{0} r^{2}}{16}(3+\mu), \\
& M_{r r}(r)=\frac{p_{0}(3+\mu)}{16}\left(r_{0}^{2}-r^{2}\right) .
\end{aligned}
$$


The bending moment at the centre of the plate is given by:

$M_{r r}(r=0)=\frac{p_{0} r_{0}^{2}(3+\mu)}{16}$

\section{Validation of the solution for simply supported circular Mindlin plate using the Ritz finite element method}

The total potential energy functional $\Pi$ for a Mindlin's first order shear deformable circular plate is given by:

$\Pi=\frac{1}{2} \iint_{A} \int_{-h / 2}^{h / 2}\left(\sigma_{r r} \varepsilon_{r r}+\sigma_{\theta \theta} \varepsilon_{\theta \theta}+\tau_{r \theta} \gamma_{r \theta}+\tau_{r z} \gamma_{r z}+\tau_{z \theta} \gamma_{z \theta}\right) d z r d r d \theta-\iint_{A} p w r d r d \theta$,

where $A$ is the cross-sectional area (domain) of the circular plate given by:

$0 \leq r \leq r_{0}, \quad 0 \leq \theta \leq 2 \pi$

From the principle of virtual displacements:

$\delta \Pi=0$,

$\iint_{A}\left[M_{r r} \frac{\partial \delta \varphi_{r}}{\partial r}+M_{r \theta}\left(\frac{1}{r} \frac{\partial \delta \varphi_{r}}{\partial \theta}+\frac{\partial \delta \varphi_{\theta}}{\partial r}-\frac{\partial \varphi_{\theta}}{r}\right)+M_{\theta \theta}\left(\frac{\delta \varphi_{r}}{r}+\frac{1}{r} \frac{\partial \delta \varphi_{\theta}}{\partial \theta}\right)\right.$
$\left.+Q_{r}\left(\delta \varphi_{r}+\frac{\partial \delta w_{0}}{\partial r}\right)+Q_{\theta}\left(\delta \varphi_{\theta}+\frac{1}{r} \frac{\partial \delta w_{0}}{\partial \theta}\right)-p \delta w_{0}\right] r d r d \theta=0$,

where:

$Q_{r}=k_{s} \int_{-h / 2}^{h / 2} \tau_{z \theta} d z$

$Q_{r}=k_{s} \int_{-h / 2}^{h / 2} \tau_{z r} d z$

The weak form of the virtual work statement is:

$$
\begin{aligned}
& \int_{0}^{2 \pi} \int_{0}^{r_{0}}\left[D\left(\frac{d}{d r} \varphi_{r}(r)+\mu \frac{\varphi_{r}(r)}{r}\right) \frac{d \delta \varphi_{r}(r)}{d r}+D\left(\mu \frac{d \varphi_{r}(r)}{d r}+\frac{\varphi_{r}(r)}{r}\right) \frac{\delta \varphi_{r}(r)}{r}\right. \\
& \left.+k_{s} G h\left(\varphi_{r}(r)+\frac{d w_{0}(r)}{d r}\right)\left(\delta \varphi_{r}(r)+\frac{d \delta w_{0}(r)}{d r}\right)-p(r) \delta w_{0}(r)\right] r d r d \theta=0 \\
& 2 \pi \int_{0}^{r_{0}}\left[D\left(\frac{d \varphi_{r}(r)}{d r}+\mu \frac{\varphi_{r}(r)}{r}\right) \frac{d \delta \varphi_{r}}{d r}+D\left(\mu \frac{d \varphi_{r}(r)}{d r}+\frac{\varphi_{r}(r)}{r}\right) \frac{\delta \varphi_{r}(r)}{r}\right. \\
& \left.\quad+k_{s} G h\left(\varphi_{r}(r)+\frac{d w_{0}(r)}{d r}\right)\left(\delta \varphi_{r}(r)+\frac{d \delta w_{0}(r)}{d r}\right)-p(r) \delta w_{0}(r)\right] r d r=0, \\
& \int_{0}^{r_{0}}\left[D\left(\frac{d \varphi_{r}}{d r}+\mu \frac{\varphi_{r}}{r}\right) \delta \frac{d \varphi_{r}}{d r}+D\left(\mu \frac{d \varphi_{r}}{d r}+\frac{\varphi_{r}}{r}\right) \frac{\delta \varphi_{r}}{r}+k_{s} G h\left(\varphi_{r}+\frac{d w_{0}}{d r}\right)\right. \\
& \left.\quad \times\left(\delta \varphi_{r}+\delta \frac{d w_{0}(r)}{d r}\right)-p(r) \delta w_{0}(r)\right] r d r=0 .
\end{aligned}
$$

Let: 
$\begin{aligned} w_{0}(r) & =\sum_{i=1}^{M} a_{i} f_{i}(r), \\ \varphi_{r}(r) & =\sum_{j=1}^{N} c_{j} g_{j}(r) .\end{aligned}$

Then:

$$
\begin{aligned}
& \frac{d \varphi_{r}(r)}{d r}=\sum_{j=1}^{N} c_{j} \frac{d g_{j}(r)}{d r} \\
& \frac{d w_{0}(r)}{d r}=\sum_{i=1}^{M} a_{i} \frac{d f_{i}(r)}{d r} \\
& \int_{0}^{r_{0}}\left[D\left(\sum c_{j} \frac{d g_{j}}{d r}+\mu \sum c_{j} g_{j}\right) \delta \sum c_{k} \frac{d g_{k}}{d r}\right. \\
& \quad+D\left(\mu \sum c_{j} \frac{d g_{j}}{d r}+\frac{1}{r} \sum c_{j} g_{j}(r)\right) \frac{1}{r} \delta \sum c_{k} g_{k} \\
& \left.\quad+k_{s} G h\left(\sum c_{j} g_{j}+\sum a_{i} \frac{d f_{i}}{d r}\right)\left(\delta \sum c_{k} g_{k}+\delta \sum a_{k} \frac{d f_{k}}{d r}\right)-p(r) \delta \sum a_{i} f_{i}\right] r d r=0
\end{aligned}
$$

Equating the coefficients of $\delta a_{i}$ and $\delta c_{i}$ to zero, and this corresponds to the requirement that $\partial \Pi=0$ with respect to $a_{i}$ and $\partial \Pi=0$ with respect to $c_{i}$ we have the following Ritz variational statements:

$$
\begin{aligned}
& \sum_{j=1}^{M}\left[k_{s} G h \int_{0}^{r_{0}} \frac{d f_{i}(r)}{d r} \frac{d f_{j}(r)}{d r} r d r\right] a_{j}+\sum_{j=1}^{M}\left[k_{s} G h \int_{0}^{r_{0}} \frac{d f_{i}}{d r} g_{j}(r) r d r\right] c_{j} \\
& \quad-\int_{0} p(r) f_{i}(r) r d r=0, \\
& k_{S} G h \sum_{j=1}^{M}\left[\int_{0}^{r_{0}} g_{k}(r) \frac{d f_{j}(r)}{d r} r d r\right] a_{j}+\sum_{j=1}^{N}\left\{k_{s} G h \int_{0}^{r_{0}} g_{k}(r) g_{j}(r) r d r\right. \\
& \left.+D \int_{0}^{r_{0}}\left[\frac{d g_{k}(r)}{d r} \frac{d g_{j}(r)}{d r}+\frac{\mu}{r}\left(g_{k}(r) \frac{d g_{j}(r)}{d r}+\frac{d g_{k}}{d r} g_{j}(r)\right)+\frac{1}{r^{2}} g_{k}(r) g_{j}(r)\right] r d r\right\} c_{j}=0,
\end{aligned}
$$

where $k=1,2, \ldots, N$.

In matrix form, the Ritz variational equations are:

$\left[\begin{array}{ll}{\left[X_{i j}\right]} & {\left[Y_{i j}\right]} \\ {\left[Y_{i j}\right]^{T}} & {\left[Z_{i j}\right]}\end{array}\right]\left(\begin{array}{l}{\left[a_{i}\right]} \\ {\left[c_{j}\right]}\end{array}\right)=\left\{\begin{array}{c}P_{i} \\ 0\end{array}\right\}$,

in which:

$$
\begin{aligned}
& {\left[X_{i j}\right]=k_{s} G h \int_{0}^{r_{0}} \frac{d f_{i}(r)}{d r} \frac{d f_{j}(r)}{d r} r d r,} \\
& {\left[X_{i j}\right]=k_{s} G h \int_{0}^{r_{0}} f_{i}^{\prime}(r) f_{j}^{\prime}(r) r d r}
\end{aligned}
$$




$$
\begin{aligned}
{\left[Y_{i j}\right] } & =k_{s} G h \int_{0}^{r_{0}} \frac{d f_{i}(r)}{d r} g_{j}(r) r d r, \\
{\left[Y_{i j}\right] } & =k_{s} G h \int_{0}^{r_{0}} f_{i}^{\prime}(r) g_{j}(r) r d r, \\
{\left[P_{i}\right] } & =\int_{0}^{r_{0}} p(r) f_{i}(r) r d r \\
{\left[Z_{k j}\right] } & =D \int_{0}^{r_{0}}\left[\frac{d g_{k}(r)}{d r} \frac{d g_{j}(r)}{d r}+\frac{\mu}{r}\left(g_{k}(r) \frac{d g_{j}(r)}{d r}+\frac{d g_{k}(r)}{d r} g_{j}(r)+\frac{1}{r^{2}} g_{k}(r) g_{j}(r)\right)\right] r d r \\
+ & k_{s} G h \int_{0}^{r_{0}} g_{k}(r) g_{j}(r) r d r .
\end{aligned}
$$

\subsubsection{Simply supported solid first order shear deformable circular plate}

Shape functions for simply supported solid first order shear deformable circular plates are:

$$
\begin{aligned}
& f_{1}(r)=1-\frac{r}{r_{0}}, \\
& f_{2}(r)=1-\frac{r^{2}}{r_{0}^{2}}, \\
& g_{1}(r)=\frac{r}{r_{0}},
\end{aligned}
$$

where $f_{1}, f_{2}$ and $g_{1}$ satisfy the essential boundary conditions:

$$
\begin{aligned}
& w_{0}\left(r=r_{0}\right)=0, \\
& \varphi_{r}(r=0)=0, \\
& X_{11}=k_{s} G h \int_{0}^{r_{0}} f_{1}^{\prime}(r) f_{1}^{\prime}(r) r d r, \\
& f_{1}^{\prime}(r)=\left(-\frac{1}{r_{0}}\right) \\
& X_{11}=k_{s} G h \int_{0}^{r_{0}}\left(-\frac{1}{r_{0}}\right)\left(-\frac{1}{r_{0}}\right) r d r=\frac{k_{s} G h}{r_{0}^{2}} \int_{0}^{r_{0}} r d r= \\
& X_{11}=\frac{k_{s} G h}{r_{0}^{2}}\left[\frac{r^{2}}{2}\right]_{0}^{r_{0}}=\frac{k_{s} G h}{r_{0}^{2}} \frac{r_{0}^{2}}{2}=\frac{k_{s} G h}{2}, \\
& X_{12}=k_{s} G h \int_{0}^{r_{0}} f_{1}^{\prime}(r) f_{2}^{\prime}(r) r d r, \\
& X_{12}=k_{s} G h \int_{0}^{r_{0}}\left(-\frac{1}{r_{0}}\right)\left(-\frac{2 r}{r_{0}^{2}}\right) r d r=\frac{2 k_{s} G h}{r_{0}^{3}} \int_{0}^{r_{0}} r^{2} d r, \\
& X_{12}=\frac{2 k_{s} G h}{r_{0}^{3}}\left[\frac{r^{3}}{3}\right]_{0}^{r_{0}}=\frac{2 k_{s} G h}{r_{0}^{3}} \frac{r_{0}^{3}}{3}=\frac{2 k_{s} G h}{3}, \\
& Y_{11}=k_{s} G h \int_{0}^{r_{0}}\left(-\frac{1}{r_{0}}\right)\left(\frac{r}{r_{0}}\right) r d r, \\
& Y_{11}=-\frac{k_{s} G h}{r_{0}^{2}} \int_{0}^{r_{0}} r^{2} d r=-\frac{k_{s} G h}{r_{0}^{2}}\left[\frac{r^{3}}{3}\right]_{0}^{r_{0}}=-\frac{k_{s} G h}{r_{0}^{2}} \frac{r_{0}^{3}}{3}=-\frac{k_{s} G h r_{0}}{3}, \\
& Y_{21}=k_{s} G h \int_{2}^{\prime} g_{1} r d r,
\end{aligned}
$$




$$
\begin{aligned}
& Y_{21}=k_{s} G h \int_{0}^{r_{0}}\left(-\frac{2 r}{r_{0}^{2}}\right) \frac{r}{r_{0}} r d r \\
& Y_{21}=-\frac{2 k_{s} G h}{r_{0}^{3}} \int_{0}^{r_{0}} r^{3} d r=-\frac{2 k_{s} G h}{r_{0}^{3}}\left[\frac{r^{4}}{4}\right]_{0}^{r_{0}}=-\frac{2 k_{s} G h}{r_{0}^{3}}\left(\frac{r_{0}^{4}}{4}\right)=-\frac{k_{s} G h r_{0}}{2}, \\
& X_{21}=k_{s} G h \int_{0}^{r_{0}} f_{2}^{\prime}(r) f_{1}^{\prime} r d r \\
& X_{21}=k_{S} G h \int_{0}^{r_{0}}\left(-\frac{2 r}{r_{0}^{2}}\right)\left(-\frac{1}{r_{0}}\right) r d r=\frac{2 k_{s} G h}{r_{0}^{3}} \int_{0}^{r_{0}} r^{2} d r=\frac{2 k_{s} G h}{r_{0}^{3}}\left[\frac{r^{3}}{3}\right]_{0}^{r_{0}} \text {, } \\
& X_{21}=\frac{2 k_{s} G h}{r_{0}^{3}}\left(\frac{r_{0}^{3}}{3}\right)=\frac{2}{3} k_{s} G h \text {, } \\
& X_{22}=k_{s} G h \int_{0}^{r_{0}} f_{2}^{\prime}(r) f_{2}^{\prime}(r) r d r \text {, } \\
& X_{22}=k_{s} G h \int_{0}^{r_{0}}\left(-\frac{2 r}{r_{0}^{2}}\right)\left(-\frac{2 r}{r_{0}^{2}}\right) r d r \text {, } \\
& X_{22}=\frac{4 k_{s} G h}{r_{0}^{4}} \int_{0}^{r_{0}} r^{3} d r=\frac{4 k_{s} G h}{r_{0}^{4}}\left(\frac{r^{4}}{4}\right)_{0}^{r_{0}}=\frac{4 k_{s} G h}{r_{0}^{4}} \frac{r_{0}^{4}}{4}=k_{s} G h \text {, } \\
& Z=D \int_{0}^{r_{0}}\left(\left(\frac{1}{r_{0}}\right)\left(\frac{1}{r_{0}}\right)+\frac{\mu}{r}\left(\frac{r}{r_{0}} \frac{1}{r_{0}}+\frac{1}{r_{0}} \frac{r}{r_{0}}\right)+\frac{1}{r^{2}} \frac{r}{r_{0}} \frac{r}{r_{0}}\right) r d r+k_{s} G h \int_{0}^{r_{0}}\left(\frac{r}{r_{0}}\right)\left(\frac{r}{r_{0}}\right) r d r, \\
& Z=D \int_{0}^{r_{0}}\left(\frac{1}{r_{0}^{2}}+\frac{\mu}{r}\left(\frac{2 r}{r_{0}^{2}}\right)+\frac{1}{r_{0}^{2}}\right) r d r+\frac{k_{s} G h}{r_{0}^{2}} \int_{0}^{r_{0}} r^{3} d r \text {, } \\
& Z=\frac{D}{r_{0}^{2}} \int_{0}^{r_{0}}(2+2 \mu) r d r+\frac{k_{s} G h}{r_{0}^{2}} \int_{0}^{r_{0}} r^{3} d r, \\
& Z=\frac{(2+2 \mu) D}{r_{0}^{2}} \int_{0}^{r_{0}} r d r+\frac{k_{s} G h}{r_{0}^{2}} \int_{0}^{r_{0}} r^{3} d r, \\
& Z=\frac{(2+2 \mu) D}{r_{0}^{2}}\left[\frac{r^{2}}{2}\right]_{0}^{r_{0}}+\frac{k_{s} G h}{r_{0}^{2}}\left[\frac{r^{4}}{4}\right]_{0}^{r_{0}}, \\
& Z=(1+\mu) D+\frac{k_{s} G h}{r_{0}^{2}} \frac{r_{0}^{4}}{4}=(1+\mu) D+\frac{k_{s} G h}{4} r_{0}^{2} \text {, } \\
& Z=\frac{k_{s} G h}{12}\left(3 r_{0}^{2}+\frac{12(1+\mu) D}{k_{s} G h}\right) \text {, } \\
& P_{1}=\int_{0}^{r_{0}} p_{0}\left(1-\frac{r}{r_{0}}\right) r d r=p_{0} \int_{0}^{r_{0}}\left(1-\frac{r}{r_{0}}\right) r d r=p_{0} \int_{0}^{r_{0}}\left(r-\frac{r^{2}}{r_{0}}\right) d r \text {, } \\
& P_{1}=p_{0}\left[\frac{r^{2}}{2}-\frac{r^{3}}{3 r_{0}}\right]_{0}^{r_{0}}=p_{0}\left(\frac{r_{0}^{2}}{2}-\frac{r_{0}^{3}}{3 r_{0}}\right) \text {, } \\
& P_{1}=p_{0}\left(\frac{r_{0}^{2}}{2}-\frac{r_{0}^{2}}{3}\right)=\frac{p_{0} r_{0}^{2}}{6} \text {, } \\
& P_{2}=\int_{0}^{r_{0}} p_{0}\left(1-\frac{r^{2}}{r_{0}^{2}}\right) r d r=p_{0} \int_{0}^{r_{0}}\left(r-\frac{r^{3}}{r_{0}^{2}}\right) d r, \\
& P_{2}=p_{0}\left[\frac{r^{2}}{2}-\frac{r^{4}}{4 r_{0}^{2}}\right]_{0}^{r_{0}}=p_{0}\left(\frac{r_{0}^{2}}{2}-\frac{r_{0}^{2}}{4}\right)=\frac{p_{0} r_{0}^{2}}{4} \text {. }
\end{aligned}
$$

The Ritz variational equations become in matrix form: 


$$
\begin{gathered}
\frac{k_{s} G h}{12}\left[\begin{array}{ccc}
6 & 8 & -4 r_{0} \\
8 & 12 & -6 r_{0} \\
-4 r_{0} & -6 r_{0} & \left(3 r_{0}^{2}+\frac{12(1+\mu) D}{k_{s} G h}\right)
\end{array}\right]\left(\begin{array}{l}
a_{1} \\
a_{2} \\
c_{1}
\end{array}\right)=\frac{p_{0} r_{0}^{2}}{12}\left(\begin{array}{l}
2 \\
3 \\
0
\end{array}\right), \\
\left(\begin{array}{ccc}
6 & 8 & -4 r_{0} \\
8 & 12 & -6 r_{0} \\
-4 r_{0} & -6 r_{0} & \left(3 r_{0}^{2}+\frac{12(1+\mu) D}{k_{s} G h}\right)
\end{array}\right)\left(\begin{array}{l}
a_{1} \\
a_{2} \\
c_{1}
\end{array}\right)=\frac{p_{0} r_{0}^{2}}{k_{s} G h}\left(\begin{array}{l}
2 \\
3 \\
0
\end{array}\right) .
\end{gathered}
$$

The undetermined displacement parameters are:

$a_{1}=0$,

$a_{2}=\frac{p_{0} r_{0}^{2}}{24}\left(\frac{4}{k_{s} G h}+\frac{r_{0}^{2}}{D(1+\mu)}\right)$

$c_{1}=\frac{2 p_{0} r_{0}^{3}}{3 D(1+\mu)}$.

The Ritz variational solution is:

$$
\begin{aligned}
& w_{2}(r)=\frac{p_{0} r_{0}^{4}}{24 D(1+\mu)}\left(1-\frac{r^{2}}{r_{0}^{2}}\right)+\frac{p_{0} r_{0}^{2}}{6 k_{s} G h}\left(1-\frac{r^{2}}{r_{0}^{2}}\right), \\
& \varphi_{1}(r)=\frac{2 p_{0} r_{0}^{3}}{3 D(1+\mu)}\left(\frac{r}{r_{0}}\right) .
\end{aligned}
$$

The Ritz variational solutions are only approximate.

\subsection{Validation of solution for clamped circular Mindlin plate}

The solutions obtained in this study for Mindlin's first order shear deformable solid circular plate with clamped edge and under uniform transverse load distribution are exactly the same as solutions obtained by Ike et al. [31] who used the method of direct integration of the biharmonic equation for circular Mindlin plates, and the enforcement of appropriate boundary conditions.

\section{Discussion}

In this work, the governing partial differential equations of equilibrium for axially symmetric flexural problems of first order shear deformable circular plates were solved analytically to obtain closed form mathematical solutions for the unknown generalised displacements $w_{0}(r)$ and $\varphi_{r}(r)$. The governing equations, Eqs. (40) and (41), for axially symmetric first order shear deformable (FSD) circular plate problems were first expressed in terms of the unknown generalised displacements with the aid of the differential equations relating the stress resultants and the generalised displacements Eqs. (42-44). The resulting differential expressions, now in variable separable form were integrated to obtain the general solutions for the unknown displacements as Eqs. (50) and (58). The general solution for the rotation function $\varphi_{r}(r)$ contained three unknown constants of integration; while the general solutions for the deflection $w_{0}(r)$ contained four unknown constants of integration. Mathematical solutions for $w_{0}(r)$ and $\varphi_{r}(r)$ were then sought for three specific cases.

For first order shear deformable solid circular plate with clamped edge and under uniformly distributed transverse load over the entire plate domain, boundedness conditions for the rotation, and the need for the shear force at the centre to vanish were used to find two constants of integration as Eqs. (59) and (60). The boundary conditions at the clamped edge - Eqs. (61) and 
(62) - were used to obtain the other two constants of integration as Eqs. (68) and (69). The deflection function was thus obtained as Eq. (70) and the rotation function as Eq. (72). It was observed that the deflection function could be decomposed into a flexural component, given by Eq. (74) and a shear component given by Eq. (75). The maximum deflection was found to occur at the centre of the plate, in line with the demands of the symmetry of the plate and the loading. The maximum deflection was found as Eqs. (76) or (77). For the particular case where $k_{s}=5 / 6$ and $\mu=0.30$, the maximum deflection was obtained as Eq. (78). The maximum deflection of first order shear deformable circular plate with clamped edge for the case of uniformly distributed transverse load are shown in Table 1 for various values of the ratio of the plate thickness to the radius $\left(h / r_{0}\right)$. The difference between the maximum deflection values obtained using the first order shear deformable circular plate with clamped edge for the case of uniformly distributed load were calculated for varying ratios of $h / r_{0}$ and shown in Table 2 . An examination of Table 2 shows that the contribution of shear deformation to the overall bending deformation of the plate increases as the plate thickness increases. For thin plates, the difference between the maximum transverse deflection obtained using the first order shear deformable circular plate and the classical Kirchhoff-Love circular thin plate theory is insignificant. However, as the plate thickness increases, the difference becomes significant.

For the case of first order shear deformable solid circular plate with clamped edge and point load $P_{0}$ applied at the centre, the mathematical solutions for $w_{0}(r)$ and $\varphi_{r}(r)$ were obtained as Eqs. (91) and (92), respectively. The deflection $w_{0}(r)$ in the first order shear deformable circular plate was found to be decomposed into two components, flexural component, and shear component, where the flexural component corresponded to the solution for the Kirchhoff-Love circular thin plate.

It is also observed that:

$\varphi(r)=\frac{-d w_{0}^{f}(r)}{d r}$

It is observed that the shear component of the deflection is singular and undefined at $r=0$, the point of application of the point load $P_{0}$. It is thus mathematically impossible to determine the maximum deflection since the deflection is singular at the centre of the plate where $r=0$.

For the case of first order shear deformable solid circular plate with simply supported edge and under uniformly distributed transverse load over the entire plate region, the general solutions for $w_{0}(r)$ and $\varphi_{r}(r)$ were obtained as Eqs. (110) and (111) respectively. The deflection $w_{0}(r)$ was observed to be decomposed into two components; a flexural component and shear component. The maximum deflection was found to occur at the plate centre, in line with the symmetrical nature of the problem. The maximum deflection was obtained as Eqs. (113) or (114). The maximum deflection was also found in terms of the ratio $h / r_{0}$ as Eq. (115). The maximum rotation was found to occur at the plate edge and was obtained as Equation (116). The bending moment distribution $M_{r r}(r)$ was obtained as Eq. (119). Maximum bending moment $M_{r r m a x}$ was found to occur at the centre of the plate and was obtained as Eq. (120).

The significant contributions of the present study are:

(i) Closed form mathematical solutions were obtained for the general case of Mindlin's first order shear deformable circular plate subject to an arbitrary distribution of transverse load as Eqs. (50) and (58). The solutions were derived from first principles, and were derived to satisfy the governing equations of equilibrium at all points on the solution domain, which is the two dimensional domain of the circular plate $0 \leq r \leq r_{0}, 0 \leq \theta \leq 2 \pi$.

(ii) Mathematical solutions were derived from the general solution for the particular cases of clamped edge and uniformly distributed load as Eqs. (70) and (72). The solutions were found to satisfy the boundary conditions and were derived such that all points on the circular plate domain satisfied the governing equations. For point load at the centre, the mathematical solutions were 
found from the general solution as Eqs. (92) and (93).

(iii) Mathematical solutions were similarly derived from the general solution for the particular case of simply supported edge and uniformly distributed load as Eqs. (110) and (111).

\section{Conclusions}

The following conclusions are made from the study:

(i) The partial differential equations of equilibrium of axisymmetrically loaded first order shear deformable circular plates have been solved mathematically using the method of separation of variables.

(ii) The general solutions obtained for the transverse deflection $w_{0}(r)$ and the rotation functions for any arbitrary axisymmetric load distribution contained four constants of integration, which are determined for specific cases of edge supports and transverse loads by the application of the appropriate boundary conditions.

(iii) The general solution obtained for the deflection of first order shear deformable solid circular plates fixed at the edge $r=r_{0}$ was decomposable into the flexural component and a shear component. The flexural component of the deflection in this case was exactly the same as the deflection expression for a Kirchhoff-Love circular plate of the same radius. The contribution of the shear strength component to the bending deformation increased significantly with increase in the ratio of plate thickness to the radius $\left(h / r_{0}\right)$.

(iv) The shear force distribution for first order shear deformable solid circular plates vanished when:

$\varphi_{r}=\frac{d w_{0}(r)}{d r}$.

(v) The deflections obtained for first order shear deformable solid circular plates with clamped edge $\left(r=r_{0}\right)$ and subject to a point load $P_{0}$ applied at the centre was decomposable into a flexural component and a shear component. The deflections in this case also increased significantly with significant increase in the ratio of the plate thickness to the radius $\left(h / r_{0}\right)$.

(vi) For first order shear deformable solid circular plates with simply supported edge $\left(r=r_{0}\right)$ and subject to uniformly distributed transverse load, the solution obtained for deflection was decomposed into a flexural component and a shear component. The deflection increased significantly with increase in the ratio of the plate thickness to the radius $\left(h / r_{0}\right)$. The flexural component of the bending deflection was found to be exactly identical with the deflections obtained using the Kirchhoff-Love theory for the circular plate with simply supported edge $\left(r=r_{0}\right)$.

(vii) For all the cases considered the maximum deflection was found to occur at the plate centre; in line with the requirements of symmetry of the problems. The expressions for the maximum deflection in all the three cases were decomposable into flexural component and shear component.

(viii) For first order shear deformable solid circular plates with simply supported edge $\left(r=r_{0}\right)$ the maximum rotation was found to occur at the simply supported edge $\left(r=r_{0}\right)$.

(ix) Mathematical (closed form) expressions were obtained for the generalised displacements $w_{0}(r)$ and $\varphi_{r}(r)$ for any given load distribution $p(r)$ and any support (restraint) condition.

(x) Mathematical (closed form) expressions were obtained for the unknown generalised displacements in the boundary value problem for specific cases of fixed edge and simply supported edge and for uniformly distributed transverse load on the plate domain and for point load applied at the centre. 


\section{References}

[1] Civalek O., Oztuk B. Discrete singular convolution algorithm for non-linear transient response of circular plates resting on Winkler-Pasternak foundations with different types of dynamic loading. Indian Journal of Engineering and Material Sciences, Vol. 16, 2009, p. 259-268.

[2] Steele C. R., Balach C. D. Introduction to the theory of plates. Stanford University, https//web.stanford.edu/ chasst/Course $\% 20$ Notes/Introduction $\% 20$ to $\% 2$.

[3] Timoshenko S., Woinowsky Krieger S. Theory of Plates and Shells. 2nd Edition, Tata McGraw Hill, 2010.

[4] Ventsel E., Krauthammer T. Thin Plates and Shells: Theory, Analysis and Applications. Marcel Dekker Inc., USA, 2004.

[5] Szilard R. Theory and Analysis of Plates: Classical and Numerical Methods. Prentice Hall, Upper Saddle River, New Jersey, 2004.

[6] Chandrashekhara K. Theory of Plates. Universities Press, India, 2011.

[7] Megson T. H. G. An Introduction to Aircraft Structural Analysis. Elsevier Armsterdam Butterworth - Heinemann USA, 2010.

[8] Birman Victor Plate Structures: Solid Mechanics and Its Applications. Springer, New York, 2011.

[9] Kirstein A. F., Woolley R. M. Symmetrical bending of thin circular elastic plates on equally spaced point support. Journal of Research of the National Bureau of Standards - C Engineering and Instrumentation, Vol. 71, Issue 1, 1967, p. 1-10.

[10] Timoshenko S. P., Goodier J. M. Theory of Elasticity. Third Edition, McGraw Hill, New York, 1970.

[11] Sokolnikoff I. S. Mathematical Theory of Elasticity. Second Edition, McGraw Hill, New York, 1956.

[12] Hazel A. MATH 350211: Elasticity. University of Manchester, http://www.maths.manchester.ac.uk/ ahazel/MATH35021/MATH35021.html.

[13] Sadd M. H. Elasticity Theory, Application and Numerics. Elsevier Academic Press, Armsterdam, 2014.

[14] Barber J. R. Elasticity. Third Revised Edition, Springer Science and Business Media Derdrecht, The Netherlands, 2010.

[15] Kirchhoff G. R. Uber das Gleichgewicht und die Bewegung einer Elastischen Schelbe. Journal Für Die Reine Und Angewandte Mathematik, Vol. 1850, Issue 40, 1850, p. 51-88.

[16] Kapadiya H. M., Patel A. D. Review of bending solutions of thin plates. International Journal of Scientific Research and Development, Vol. 3, Issue 3, 2015, p. 1709-1712.

[17] Balasubramanian Ashwin Plate Analysis with Different Geometrics and Arbitrary Boundary Conditions. M.Sc. Thesis, Mechanical Engineering Faculty of Graduate School, The University of Texas at Arlington, 2011.

[18] Gujar P. S., Ladhane K. B. Bending analysis of simply supported and clamped circular plate. SSRG International Journal of Civil Engineering, Vol. 2, Issue 5, 2015, p. 69-75.

[19] Mindlin R. D. Influence of rotary inertia and shear on flexural motions of isotropic elastic plates. Journal of Applied Mechanics, Vol. 18, Issue 1, 1951, p. 31-38.

[20] Reissner E. On the theory of bending of elastic plates. Journal of Mathematics and Physics, Vol. 23, 1944, p. 184-191.

[21] Reissner E. The effect of transverse shear deformation on the bending of elastic plates. Journal of Applied Mechanics, Vol. 12, 1945, p. 69-77.

[22] Reissner E. A twelfth order theory of transverse bending of transverse isotropic plates. Journal of Applied Mathematics and Mechanics, Vol. 63, 1983, p. 285-289.

[23] Dickey R. W. Nonlinear bending of circular plates. Journal of Applied Mathematics., Vol. 30, Issue 1, 1976, p. 1-9.

[24] Shimpi R. P. Refined plate theory and its variants. AIAA Journal, Vol. 40, Issue 1, 2002, p. 137-146.

[25] Shimpi R. P., Patel H. G. A two variable refined plate theory for orthotropic plate analysis. International Journal of Solids and Structures, Vol. 43, Issues 22-23, 2006, p. 6783-6799.

[26] Levinson M. An accurate simple theory of statics and dynamics of elastic plates. Mechanics Research Communications, Vol. 7, 1980, p. 343-350.

[27] Reddy J. N. A simple higher order theory for laminated composite plates. Journal of Applied Mechanics, Vol. 51, 1984, p. 745-752.

[28] Reddy J. N. A refined non-linear theory of plates with transverse shear deformation. International Journal of Solids and Structures, Vol. 20, 1984, p. 881-896. 
[29] Suetake Y. Plate bending analysis using a modified plate theory. CMES, Vol. 11, Issue 3, 2006, p. 103-110.

[30] Ike C. C. Equilibrium approach in the derivation of differential equations for homogeneous isotropic Mindlin plates. Nigerian Journal of Technology, Vol. 36, Issue 2, 2017, p. 346-350.

[31] Ike C. C., Nwoji C. U., Ofondu I. O. Variational formulation of Mindlin plate equation and solution for deflections of clamped Mindlin plate. International Journal for Research in Applied Sciences and Engineering Technology, Vol. 5, Issue 1, 2017, p. 340-353.

[32] Wang Yun, Xu Rongqiao, Ding Haojiang Three dimensional solution of axisymmetric bending of functionally graded circular plates. Composite Structures, Vol. 92, 2010, p. 1683-1693.

[33] Mekalke G. C., Kavade M. V., Deshpande S. S. Analysis of a plate with a circular hole by FEM. Journal of Mechanical and Civil Engineering, 2012, p. 25-30.

[34] Gujar P. S., Pendhari S. S. Static bending analysis of an isotropic circular plate using finite element method. International Journal of Modern Trends in Engineering and Research, Vol. 3, Issue 4, 2016, p. 491-497.

[35] Aliabadi M. H., Wen P. H. Boundary Element Methods in Engineering and Sciences. Computational and Experimental Methods in Structures, Vol. 4, Imperial College Press, London, 2011.

[36] Tseng W. D., Tarn J. Q., Tung L. Y., Liang W. Y. Free vibration of circular plates with various edge boundary conditions by $3 \mathrm{D}$ elasticity theory. Journal of Robotics and Mechanical Engineering Research, Vol. 1, Issue 2, 2015, p. 28-35.

[37] Sader J. E., White L. Theoretical analysis of the static deflection of plates for atomic force microscope applications. Journal of Applied Physics, Vol. 74, Issues 1, 1993, https://doi.org/10.1063/1.354137.

[38] Karamanlidis D., The Le H., Atlure S. N. Mixed finite element models for plate bending analysis. a new element and its applications. Computers and Structures, Vol. 19, Issue 4, 1984, p. 565-581. 